\title{
Effect of Cognitive Distraction on Physiological Measures and Driving Performance in Traditional and Mixed Traffic Environments
}

\author{
Qiang Hua $\mathbb{D}$, ${ }^{1}$ Lisheng Jin $\mathbb{D},{ }^{1,2}$ Yuying Jiang $\mathbb{C}^{3},{ }^{3}$ Baicang Guo $\mathbb{D}^{1},{ }^{1}$ and Xianyi Xie $\mathbb{C}^{2}$ \\ ${ }^{1}$ Transportation College, Jilin University, Changchun 130022, China \\ ${ }^{2}$ School of Vehicle and Energy, Yanshan University, Qinhuangdao 066004, China \\ ${ }^{3}$ Department of Ophthalmology, China-Japan Union Hospital, Jilin University, Changchun 130022, China
}

Correspondence should be addressed to Lisheng Jin; jinls@ysu.edu.cn

Received 22 May 2021; Accepted 9 July 2021; Published 19 July 2021

Academic Editor: Xinqiang Chen

Copyright (c) 2021 Qiang Hua et al. This is an open access article distributed under the Creative Commons Attribution License, which permits unrestricted use, distribution, and reproduction in any medium, provided the original work is properly cited.

Distracted driving is a dominant cause of traffic accidents. In addition, with the rapid development of intelligent vehicles, mixed traffic environments are expected to become more complicated with multiple types of intelligent vehicles sharing the road, thereby increasing the opportunities for distracted driving. However, the existing research on detecting driver distraction in mixed traffic environments is limited. Therefore, in this study, we analysed the effect of cognitive distraction on the driver physiological measures and driving performance in traditional and mixed traffic environments and compared the parameters extracted in the two environments. Sixty drivers were involved in the data collection, which included normal driving and two distracting tasks while driving in a simulator. Repeated-measures analysis of variance (ANOVA) was performed to examine the effect of cognitive distraction and traffic environments on all parameters. The results indicate that the effects of the pupil diameter, standard deviations (SDs) of the horizontal and vertical fixation angles, blink frequency, speed, SD of the lane positioning (SDLP), SD of the steering wheel angle (SDSWA), and steering entropy (SE) were significant. These findings provide a theoretical foundation for identifying the most appropriate parameters to detect cognitive distraction in traditional and mixed traffic environments to help reduce traffic accidents.

\section{Introduction}

The World Health Organization claims that traffic accidents are the ninth leading cause of death, accounting for $2.2 \%$ of all deaths [1]. Statistics show that 3,477 people died in traffic accidents caused by distracted driving in the United States in 2015 [2]. Evidence indicates that driver distraction is a major cause of road traffic accidents $[3,4]$. In particular, in future mixed traffic environments, in which autonomous vehicles (AVs), connected AVs [5] (CAVs), manual vehicles (MVs), and connected MVs [6] (CMVs) will share the road, the complex driving environment is expected to involve additional factors that induce distraction. Therefore, research must be performed to identify ways to prevent distracted driving [7]. Distracted driving can impair driving performance and the ability to respond to hazardous situations. Therefore, it is particularly meaningful to study the drivers' perception abilities (driver physiological measures, subjective evaluation performance, etc.) and driving performance parameters during distracted driving and extract the parameters that are significantly different from those of normal driving to detect the distracted state of drivers $[8,9]$.

The physiological characteristics of drivers are a major aspect of distracted driving recognition that many researchers have utilized, and many studies have indicated that the effects of distracted driving on the pupil diameter, fixation angle, and blink frequency can be verified [10]; thus, these parameters can be used as cognitive distraction recognition features [11, 12]. Many previous studies illustrated that vehicle-based lateral performance parameters, such as 
the standard deviation (SD) of lane positioning (SDLP), which has been used to evaluate the lane-keeping ability during secondary task driving, can be used to detect distracted driving. Notably, distracted driving results in a significant increase in the SDLP [13], and the driver abilities in lane positioning have been noted to deteriorate under visually distracted driving $[14,15]$. The mean and SD of the lateral acceleration (SDLA) [16], which have been noted to be impaired by distracted driving, can represent unsafe driving conditions. A field study in Wuhan indicated that the lateral acceleration increases when the drivers exhibit cognitive distraction [17]. Moreover, the steering entropy (SE), which is also influenced by distracted driving, results in less smooth steering than normal driving [18]. Cell phone use has also emerged as a major contributor to distracted driving [19-21]. Several previous studies focused on steering reversal rates during distracted driving due to phone use and highlighted that a steering reversal rate of 10 represents a distracted driver state; thus, this parameter can be considered an input parameter for detecting cognitive and visual distraction [22]. Vehicle-based longitudinal performance parameters may also be relevant factors: the speed of distracted drivers is a key parameter that must be investigated. Many studies have reported that distracted drivers (visual, cognitive) compensate for their driving impairment by reducing their speed $[23,24]$. The driving speed is affected by the complexity of the driving environment, and the results indicate that the workload of drivers increases as the driving environment becomes more complex; a greater speed reduction is a compensation measure $[25,26]$.

Although many studies have attempted to identify the significant parameters that can be used to identify distracted driving, these studies were primarily based on traditional traffic environments [27]. Certain recent studies focused on drivers in the context of intelligent vehicles [28]. For example, Weaver et al. [29] conducted a comprehensive experimental study on the factors affecting the takeover performance in the process of conditional autonomous driving. The results indicated that engaging in nondriving-related tasks may lead to a deteriorated takeover performance, especially when the resource requirements overlap with those of driving tasks. Zhang et al. [30] investigated the mean takeover times from 129 studies with SAE level 2 automation or higher by using three complementary approaches. The three methods indicated that a shorter mean takeover time is associated with increased emergency situations. Notably, most of these studies focused on drivers in the context of development of intelligent vehicles, and only a few studies have been conducted on distracted driving in mixed traffic environments. At present, the traditional and mixed environments are considerably different. Thus, it is necessary to perform a comparative analysis to identify the relevant differences. Moreover, it is necessary to examine if the influence of distracted driving on the driving performance parameters and physiological measures has changed. The parameters identified in previous studies [10] can be used for distraction recognition in a traditional environment; however, it remains unclear whether these parameters can be used to identify distracted driving in a mixed environment. Furthermore, new and common parameters that can be applied for distraction recognition in a mixed environment must be clarified.

To address the abovementioned limitations in the existing research, this study considered both traditional and mixed traffic environments to analyse the physiological measures, driving performance parameters, and subjective evaluation performance of drivers under normal and distracted driving. Moreover, the effect of distracted driving on these parameters in the two traffic environments was compared to identify the significant differences, and the optimal parameters that can contribute to distraction recognition in traditional and mixed traffic environments were extracted. The experiment was conducted using a driving simulator. Normal and distracted driving tasks (two-back task and clock task) were performed in the two traffic environments. The experimental design in the traditional environment was similar to those adopted in previous studies [18]: the participant drove an MV through 24 unsignalized intersections while performing different driving tasks. In addition, the driver drove a CMV that could obtain the driving information of surrounding vehicles and assist the driver in making the optimal decisions through 24 unsignalized intersections in a mixed traffic environment. The relevant parameters were collected for 60 participants in each driving condition in the two traffic environments, and the effects of all driving tasks and traffic environments on the parameters were analysed through repeated-measures analysis of variance (ANOVA) tests. The parameters in the traditional traffic environment were compared with those in a mixed traffic environment to observe the effects of the driving environment and distracted driving on the parameters. Finally, the parameters that were adversely affected by distracted driving and significantly different from those of normal driving were extracted. The findings can provide a reference for the selection of input parameters for intelligent in-vehicle devices to detect distraction in traditional and mixed traffic environments.

This paper is organized as follows: The research methodology is presented in Section 2; specifically, the experimental environment and design scheme, as well as the parameter data to be collected are introduced. Section 3 presents the results of the systematic analysis of the effect of driving tasks on the parameters in traditional and mixed traffic environments. Finally, the parameters that can indicate cognitive distraction in traditional and mixed traffic environments are theoretically discussed, and the limitations of this paper and scope for future work are described in Section 4.

\section{Methodology}

The main framework of this study is shown in Figure 1. The parameters (driver physiological measures, subjective evaluation performance parameters, and driving performance parameters) and data analysis method were determined through a comprehensive literature review survey [22]. The traditional and mixed traffic environments were constructed in the driving simulator, and the secondary tasks were designed. Drivers were recruited to conduct the distracted driving experiment. Each driver was required to respond to a subjective 


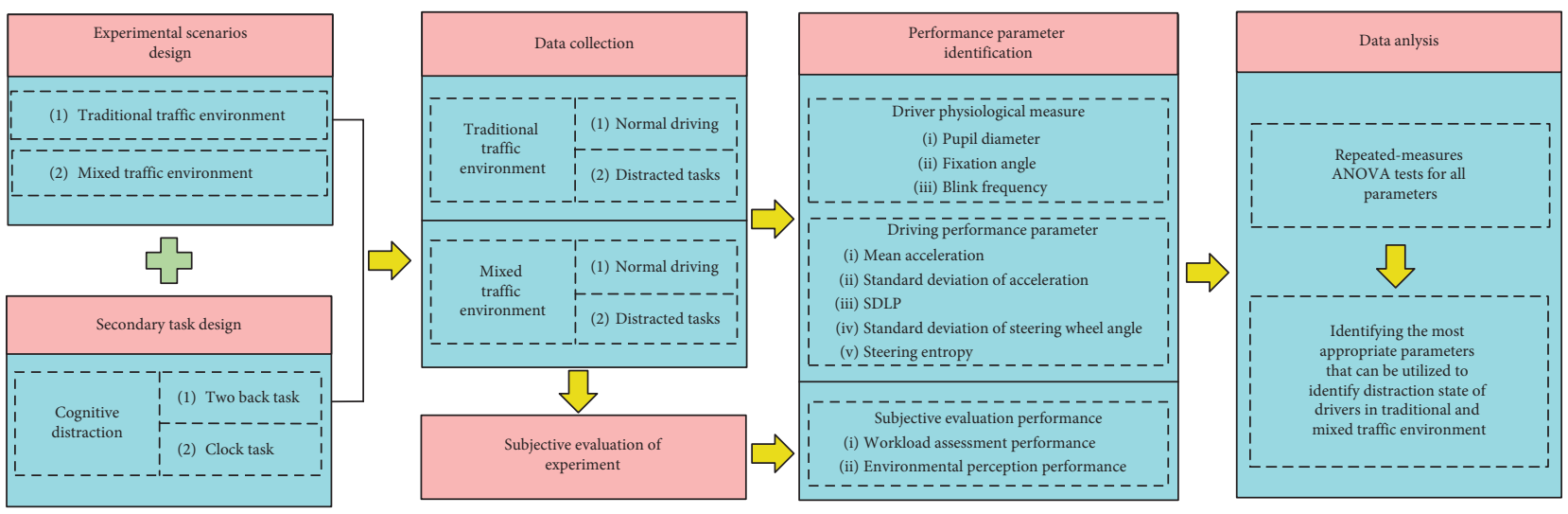

FIgURE 1: Framework of the methodology adopted for this study.

questionnaire survey after completing the driving experiment. The distracted driving data, normal driving data, and questionnaire survey data for the two traffic environments were collected and analysed through ANOVA to observe the significant effect of the parameters affected by the driving conditions and environments. The parameters that were significantly affected by distraction were extracted. Finally, a potential explanation for the result was provided. The detailed experimental process is described in the following sections.

2.1. Participants. Sixty licenced drivers were recruited for this study, and the participants conformed to the distribution of Chinese drivers based on the driver survey data of the road traffic safety research centre of the Ministry of Public Security of China in terms of the distributions of gender, age, and driving experience. The participants were divided into four age groups based on the driving experience (in years): $18 \leq$ age $\leq 25$ : mean $=4.27, \mathrm{SD}=3.12 ; 26 \leq$ age $\leq$ 35: mean $=10.7, \quad \mathrm{SD}=6.82 ; \quad 36 \leq$ age $\leq 50: \quad$ mean $=19.2$, $\mathrm{SD}=8.47$; and $60 \leq$ age: mean $=32.25, \mathrm{SD}=6.83$. The participant details are shown in Table 1.

2.2. Apparatus. The equipment used in the driving experiment included a simple driving simulator and Tobii Pro Glasses 2 eye tracker, as shown in Figure 2.

Simple driving simulator: the driving environment was established using the simulation software UC-win/Road and displayed on three 32-in LED displays. The horizontal viewing angle of the scenario display system was $120^{\circ}$, and a sound system that could provide drivers with information regarding the surrounding vehicles was used. The LOG plugin was used to collect various driving performance data that were saved in the CSV format and output. The sampling frequency was $100 \mathrm{~Hz}$. While driving, the driver was required to manipulate only the steering wheel, paddle shifters, brake pedal, and accelerator pedal to complete the driving task. The sampling rate of the Tobii Pro Glasses 2 eye tracker was $50 \mathrm{~Hz}$, and 4 eye-tracking cameras that could obtain the driver's blink frequency, fixation direction, pupil diameter, and other information were used. Python language programming was performed to ensure the synchronization of the two-channel data in time, and the interpolation method was adopted to extend the collected eye movement data to $100 \mathrm{~Hz}$ to ensure that the driving performance data and eye movement data matched in the same period.

2.3. Cognitive Tasks. The two-back task and clock task incurred a relatively large driver cognitive load and easily distracted the drivers; these tasks could be randomly arranged and repeated many times, resulting in an ideal distraction effect. Kaye et al. [31] reported that the two-back task is similar to the distraction observed on roads. The clock task mainly occupies the visuospatial working memory of the participants [32] and triggers thinking, which represents the driving behaviour occupying both mental load and visual resources. Therefore, these tasks were adopted in this study. In the two-back task, the drivers heard 10 randomized 1-digit numbers (0-9) in turn, with an interval of $2 \mathrm{~s}$ between each number. The participants were required to verbalize the two numbers preceding the number they heard. The two-back task is illustrated in Figure 3(a). In the clock task, the driving assistant randomly verbalized a time point from the prepared time list, such as 10 : 10 , and the drivers were asked to judge whether the angle between the hour hand and minute hand was an acute or obtuse angle. The time range was 1:00-12:59. The time interval for each question was $2 \mathrm{~s}$, as shown in Figure 3(b).

2.4. Driving Environment and Experimental Design. The scenarios involved unsignalized intersections of urban roads in traditional and mixed traffic environments. The roads were closed-loop two-way two-lane roads with a length of approximately $16 \mathrm{~km}$, which satisfied the Chinese traffic regulations, with a single lane width of $3.75 \mathrm{~m}$. The roads included 24 unsignalized intersections, with each intersection located in an interval of $500 \mathrm{~m}$. In the traditional environment, the vehicle driven by the participant was an MV, and the surrounding vehicles passing through the intersection were MVs. In the mixed traffic environment, the vehicle driven by the participant was a CMV, and the surrounding vehicles passing through the intersection included various intelligent vehicles. The participants drove the subject vehicle in the longitudinal lane following the 
TABLE 1: Demographic characteristics of participants.

\begin{tabular}{|c|c|c|c|c|}
\hline \multirow{2}{*}{$\begin{array}{l}\text { Demographic } \\
\text { characteristics }\end{array}$} & & \multicolumn{2}{|c|}{ Recruited drivers } & \multirow{2}{*}{ Percentage of total Chinese driver population in 2019} \\
\hline & & Quantity & Percentage & \\
\hline \multirow{2}{*}{ Gender } & Male & 42 & 70 & $70^{*}$ \\
\hline & Female & 18 & 30 & $30^{*}$ \\
\hline \multirow{4}{*}{ Age } & $18-25$ & 14 & 23.33 & $24.1^{*}$ \\
\hline & $26-35$ & 20 & 33.33 & $34.12^{*}$ \\
\hline & $36-50$ & 24 & 40 & $38.88^{*}$ \\
\hline & $>60$ & 2 & 3.3 & $2.9^{*}$ \\
\hline
\end{tabular}

* Data source: Road Traffic Safety Research Centre of the Ministry of Public Security.

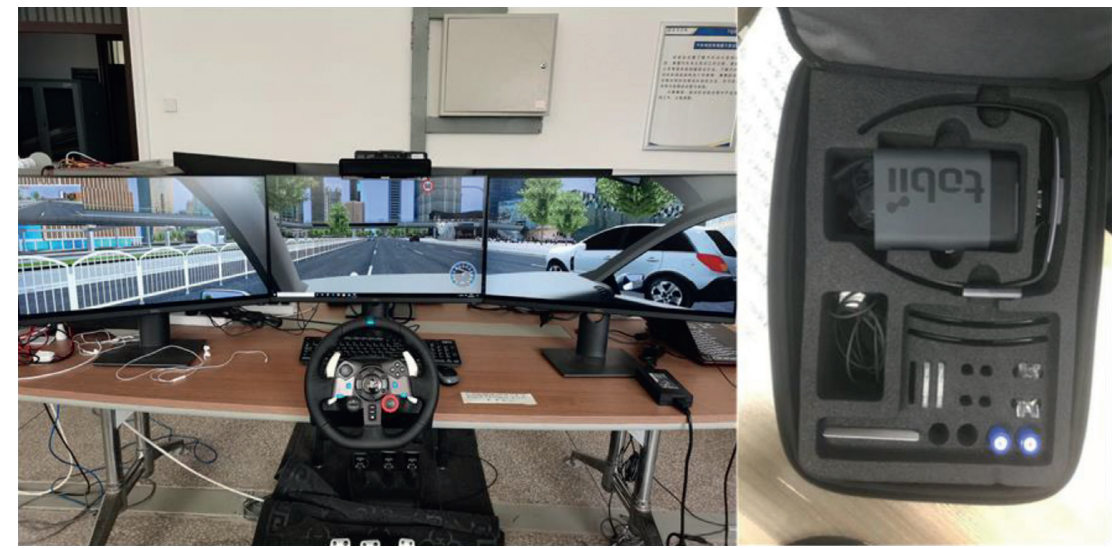

FIgURE 2: Driving simulator and eye tracker.

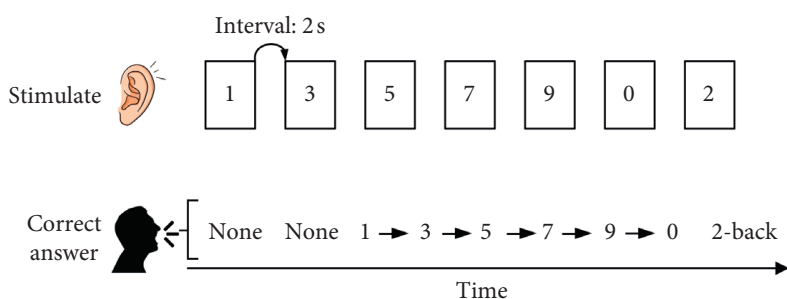

(a)

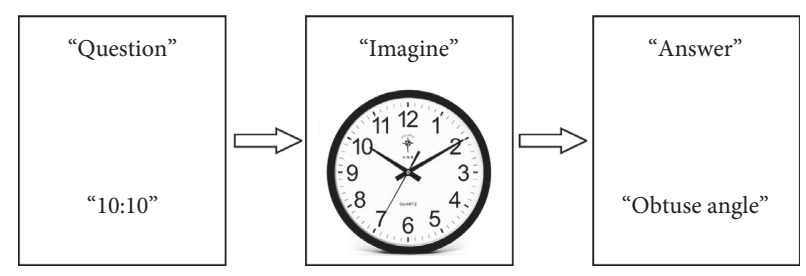

(b)

Figure 3: Illustrations of the two cognitive secondary tasks. (a) Two-back task. (b) Clock task.

traffic rule, and the posted speed limit in the scenario was $70 \mathrm{~km} / \mathrm{h}$.

When the vehicle was $120 \mathrm{~m}$ from the stop line of the intersection, the horizontal road traffic flow was triggered, in which the vehicles moved at a speed of $40 \mathrm{~km} / \mathrm{h}$ and the distance between the vehicles was randomly distributed. The participant drove the vehicle from $200 \mathrm{~m}$ before the stop line of the intersection to $20 \mathrm{~m}$ after the line, which was defined as an intersection event. While driving, the participants were required to follow the target vehicle on the longitudinal road. The speed and relative distance between the subject and target vehicles constantly varied. The top view of the experimental scenarios is shown in Figure 4. A total of 24 unsignalized intersections were present in one circle, and the driving tasks were set randomly at each intersection. The types of vehicles appearing from the lateral lanes were different in each traffic environment. The details are presented in the following sections.
2.4.1. Traditional Traffic Environment. The vehicles approaching both sides of the horizontal lane were MVs, and no communication occurred between the MVs in the lateral lane and the subject vehicle (as shown in Figure 4(a)).

2.4.2. Mixed Traffic Environment. The vehicles approaching from the left and right sides of the lateral lane were CMVs, AVs, CAVs, and MVs, and communication occurred between the connected vehicles in the horizontal lane and the subject vehicle (as shown in Figure 4(b)).

The communication information was reported to participants through the human-machine interaction interface display and voice broadcast to provide driving assistance, which included the speed of the surrounding vehicle (for instance, the speeds of the vehicle ahead (target vehicle) and that in the lateral lane) and distance from the intersection. Finally, the participant's vehicle met the vehicle in the lateral 


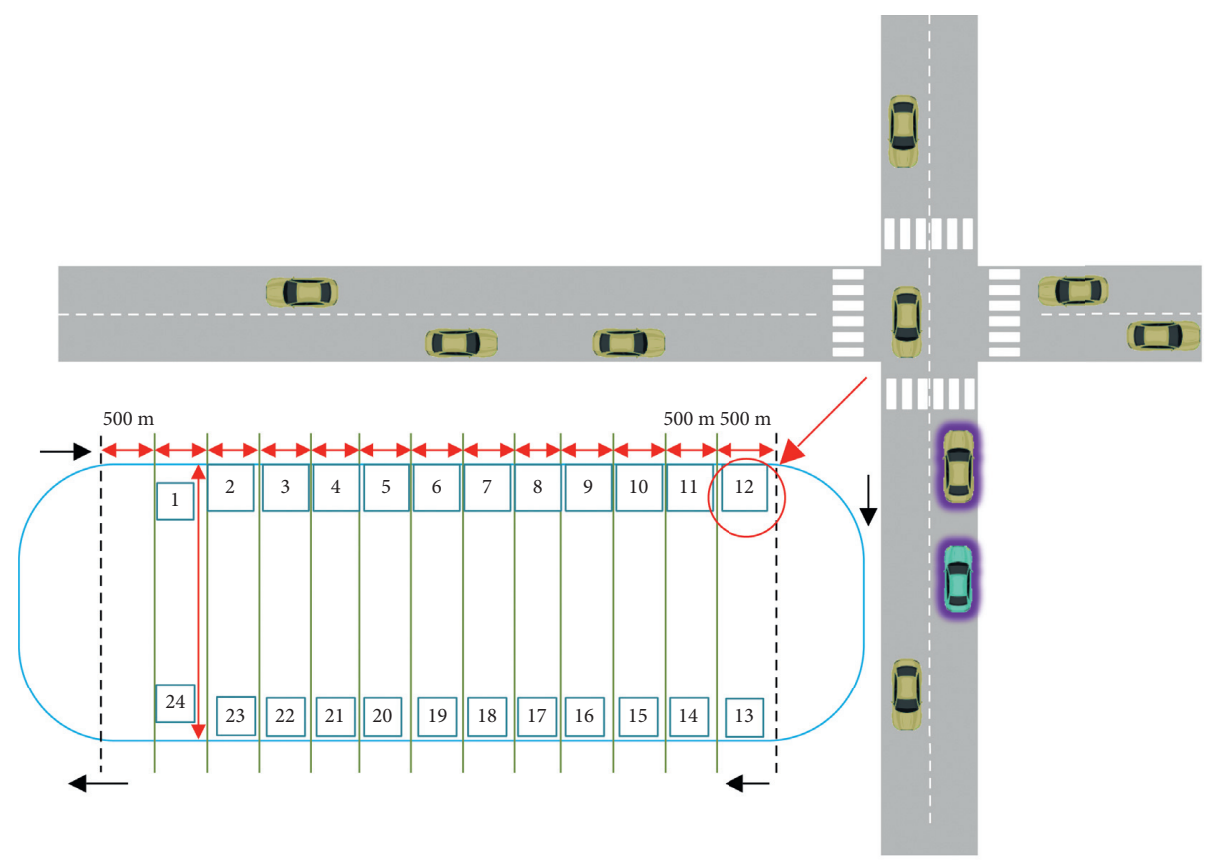

(1) Subject vehicle (MV)

(1) Target vehicle (MV)

(a)

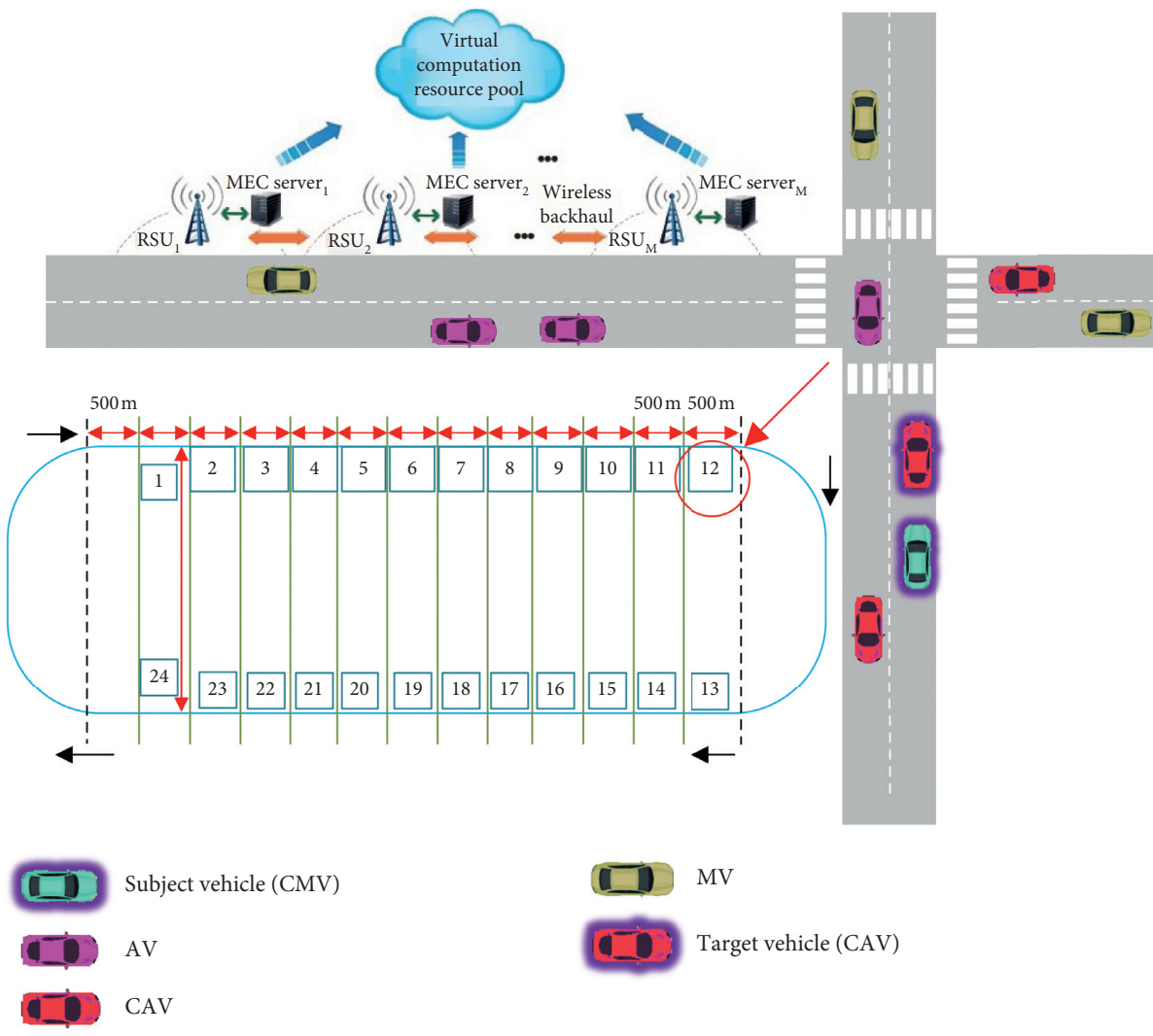

(b)

Figure 4: (a) Traditional traffic environment. (b) Mixed traffic environment. 
lane at the intersection. The driver stopped and gave way and left the intersection. When secondary tasks were assigned to the participant, the driving mode was considered to be distracted driving. In the absence of any secondary tasks, the driving task was considered to be the baseline.

2.5. Procedure. A 15 min training and practice session for both the secondary tasks and normal driving was conducted before the formal experiment to ensure that the participants were accustomed to all the experimental procedures. After the trial drive, the participants completed the formal experiment. During the experiment, no interference from other human factors occurred except for the secondary tasks, and the execution time of the secondary tasks in each event exceeded the data extraction time of each event, which ensured that the distracted driving covered the entire target event. The presentation order of the two secondary tasks was counterbalanced across participants to control the learning effects. If any problem occurred during the experiment, the drivers restarted the experiment to ensure the validity of the experimental data.

\section{Data Analysis}

\subsection{Dependent Variables}

\subsubsection{Driver Physiological Measures}

(i) Pupil diameter: the diameter of the pupil of a driver is different under different cognitive loads; thus, this parameter is a key metric used to measure the driver cognitive load (measured in metres) [10].

(ii) SD of the horizontal and vertical fixation angles: the SD of the horizontal and vertical fixation angles was used as an index to evaluate the search breadth of the viewing angle and identify the difference between the fixation angles of the driver in normal driving and distracted task driving in the two traffic environments (in degrees) [33].

(iii) Blink frequency: the blink frequency can reflect the psychological reaction of the driver to a certain extent. After the elimination of invalid data, 150 valid data samples were obtained from each driver under each driving task, and the blinking data recorded by the eye tracker were converted into the blinking frequency (in times/min).

\subsubsection{Longitudinal Control Parameter}

(i) Speed: the speed was obtained from the extracted data at each intersection.

(ii) Mean and SD of acceleration: the mean and SD of acceleration for each intersection were measured for all drivers (in $\mathrm{m} / \mathrm{s}^{2}$ ).

\subsubsection{Lateral Control Parameter}

(i) SD of lane positioning (SDLP): This parameter reflects the drivers' lane-keeping capabilities. This parameter was calculated based on a time series of historical lateral position coordinates.

(ii) Mean and SD of the steering wheel angle (SDSWA): The mean and SDSWA for each intersection were measured for all drivers (in degrees).

(iii) SE: This parameter indicates the smoothness of steering control to quantify the workload imposed on drivers [34]. SE values were obtained from a time series of historical steering angles.

\subsubsection{Subjective Evaluation Performance}

(i) Workload assessment performance: this parameter represents the overall driving burden experienced by the drivers in the driving process.

(ii) Environmental perception performance: this parameter represents the extent to which drivers can perceive roads, traffic environments, and other vehicles during the driving process.

Certain parameter data were collected from the eye tracker and driving simulator, such as the pupil diameter, fixation angle, speed, acceleration, and steering wheel angle. The values of the descriptive statistical parameters, such as the mean, SDSWA, and SE, were calculated from the measured data, as mentioned. The performances of the workload assessment and environmental perception were obtained through the participants' scoring according to the scale (see Table 2) when the formal experiment was completed.

3.2. Results. A 3 (driving task: normal driving, two-back task, clock task) $* 2$ (environment: traditional traffic environment, mixed traffic environment) repeated-measures ANOVA was conducted for each metric; the data were also checked for sphericity assumptions. If Mauchly's assumption of sphericity was violated, the Greenhouse-Geisser epsilon correction was implemented. Bonferroni's post hoc tests at a 0.05 significance level were performed if the main or any interaction effect was found to be significant. The following sections present the results obtained from the ANOVA tests of all the parameters, and the main results are summarized in Table 2.

3.2.1. Pupil Diameter. The variation in the driver's pupil diameter during the driving tasks in the two traffic environments is shown in Figures 5(a) and 5(b). The results demonstrate that, in the traditional and mixed traffic environments, the pupil diameter of the driver increases with the increases of driving load. These results are consistent with previously reported results [37]. 
TABLE 2: ANOVA tests on all parameters.

\begin{tabular}{|c|c|c|c|c|c|}
\hline Driving performance & Effect & $d f_{1}$ & $d f_{2}$ & $F$-value & $p$ value \\
\hline \multirow{3}{*}{ Pupil diameter } & Driving task & 2 & 597 & 18.32 & $p<0.001$ \\
\hline & Environment & 1 & 597 & 7.723 & $p=0.013$ \\
\hline & Driving task $*$ environment & 2 & 597 & 6.841 & $p=0.026$ \\
\hline \multirow{3}{*}{ Standard deviation of the horizontal fixation angle } & Driving task & 2 & 197 & 5.215 & $p=0.041$ \\
\hline & Environment & 1 & 197 & 3.646 & $p=0.062$ \\
\hline & Driving task $*$ environment & 2 & 197 & 5.252 & $p=0.034$ \\
\hline \multirow{3}{*}{ Standard deviation of the vertical fixation angle } & Driving task & 2 & 197 & 7.054 & $p=0.027$ \\
\hline & Environment & 1 & 197 & 6.211 & $p=0.032$ \\
\hline & Driving task $*$ environment & 2 & 197 & 1.627 & $p=0.732$ \\
\hline \multirow{3}{*}{ Blink frequency } & Driving task & 2 & 149 & 24.51 & $p<0.001$ \\
\hline & Environment & 1 & 149 & 6.726 & $p=0.036$ \\
\hline & Driving task $*$ environment & 2 & 149 & 5.493 & $p=0.017$ \\
\hline \multirow{3}{*}{ Speed } & Driving task & 2 & 714 & 16.22 & $p=0.002$ \\
\hline & Environment & 1 & 714 & 6.180 & $p=0.021$ \\
\hline & Driving task $*$ environment & 2 & 714 & 4.962 & $p=0.037$ \\
\hline \multirow{3}{*}{ Mean acceleration } & Driving task & 2 & 234 & 0.132 & $p=0.923$ \\
\hline & Environment & 1 & 234 & 0.251 & $p=0.728$ \\
\hline & Driving task $*$ environment & 2 & 234 & 0.223 & $p=0.816$ \\
\hline \multirow{3}{*}{ Standard deviation of the acceleration } & Driving task & 2 & 234 & 0.216 & $p=0.827$ \\
\hline & Environment & 1 & 234 & 0.151 & $p=0.847$ \\
\hline & Driving task $*$ environment & 2 & 234 & 0.324 & $p=0.631$ \\
\hline \multirow{3}{*}{ SDLP } & Driving task & 2 & 234 & 16.33 & $p<0.001$ \\
\hline & Environment & 1 & 234 & 2.623 & $p=0.056$ \\
\hline & Driving task $*$ environment & 2 & 234 & 0.754 & $p=0.293$ \\
\hline \multirow{3}{*}{ Mean steering wheel angle } & Driving task & 2 & 234 & 0.331 & $p=0.843$ \\
\hline & Environment & 1 & 234 & 0.275 & $p=0.756$ \\
\hline & Driving task $*$ environment & 2 & 234 & 0.312 & $p=0.871$ \\
\hline \multirow{3}{*}{ Standard deviation of the steering wheel angle } & Driving task & 2 & 234 & 20.37 & $p<0.001$ \\
\hline & Environment & 1 & 234 & 1.463 & $p=0.08$ \\
\hline & Driving task $*$ environment & 2 & 234 & 3.782 & $p=0.027$ \\
\hline \multirow{3}{*}{ Steering entropy } & Driving task & 2 & 234 & 19.56 & $p<0.001$ \\
\hline & Environment & 1 & 234 & 1.521 & $p=0.752$ \\
\hline & Driving task $*$ environment & 2 & 234 & 0.624 & $p=0.315$ \\
\hline \multirow{3}{*}{ Workload assessment performance } & Driving task & 2 & 174 & 15.78 & $p<0.001$ \\
\hline & Environment & 1 & 174 & 0.629 & $p=0.127$ \\
\hline & Driving task $*$ environment & 2 & 174 & 0.213 & $p=0.562$ \\
\hline \multirow{3}{*}{ Environmental perception performance } & Driving task & 2 & 174 & 13.29 & $p<0.001$ \\
\hline & Environment & 1 & 174 & 5.262 & $p=0.032$ \\
\hline & Driving task $*$ environment & 2 & 174 & 18.23 & $p<0.001$ \\
\hline
\end{tabular}

A 3 (task) $\times 2$ (environment) repeated-measures ANOVA was conducted on the pupil diameter results. The results indicate that the interaction effect of the driving task with the environment is significant (as shown in Figure 6(b)). Moreover, the driving task and environment have significant effects on the pupil diameter (see Table 2). Bonferroni's post hoc results show that the pupil diameter is significantly larger in the case of distracted driving than that under normal driving [38]. In addition, a significant difference exists between the clock task and two-back task. The mean values of the pupil diameter across the driving tasks are shown in Figure 6(a). The range of the fluctuation of pupil diameter in the mixed traffic environment is larger than that in the traditional traffic environment (as shown in Figures 5(a) and 5(b)), which indicates that the complexity of the mixed traffic environment is higher than that of the traditional traffic environment, resulting in a higher baseline of the overall driver level.
3.2.2. Standard Deviation (SD) of the Fixation Angle. A 2 (environment) $\times 3$ (driving task) repeated-measures ANOVA was performed on the SDs of the horizontal and vertical fixation angles, as shown in Table 2. The results indicate the presence of significant differences across the driving tasks for the mean SD of the horizontal and vertical fixation angles, as shown in Figures 7(a) and 7(b). The post hoc analysis highlights that distracted driving results in lower SDs of both the horizontal and vertical fixation angles compared to those in normal driving, which is consistent with the results of a previous study [39]. This aspect can be attributed to the driver's fixation point coverage being distributed widely while driving normally. However, the driver's field of view becomes narrower and relatively concentrated under distracted driving, in accordance with the previously reported results $[40,41]$. The horizontal and vertical fixation angles in the clock task are the lowest, 


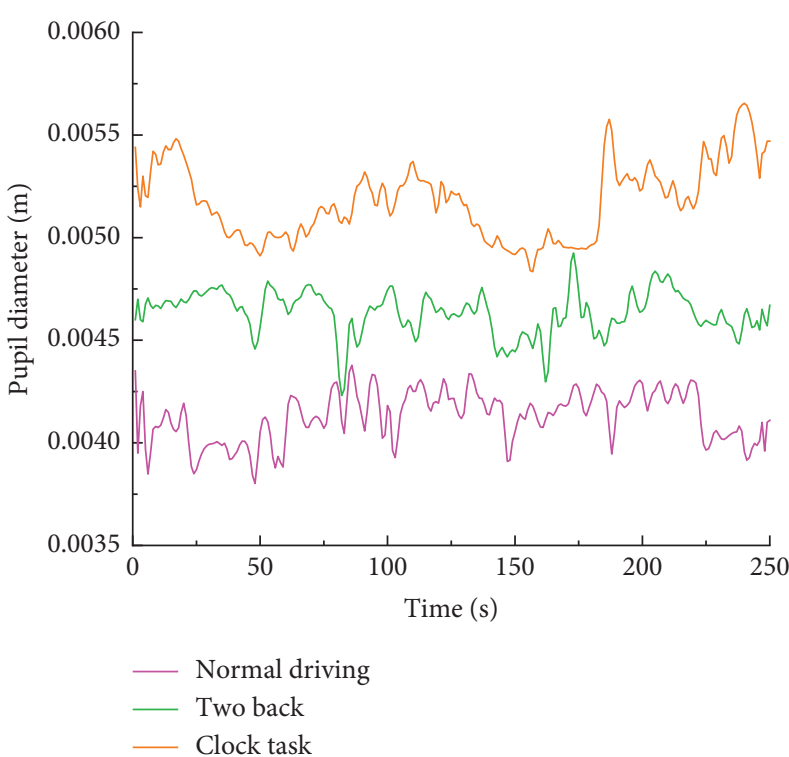

(a)

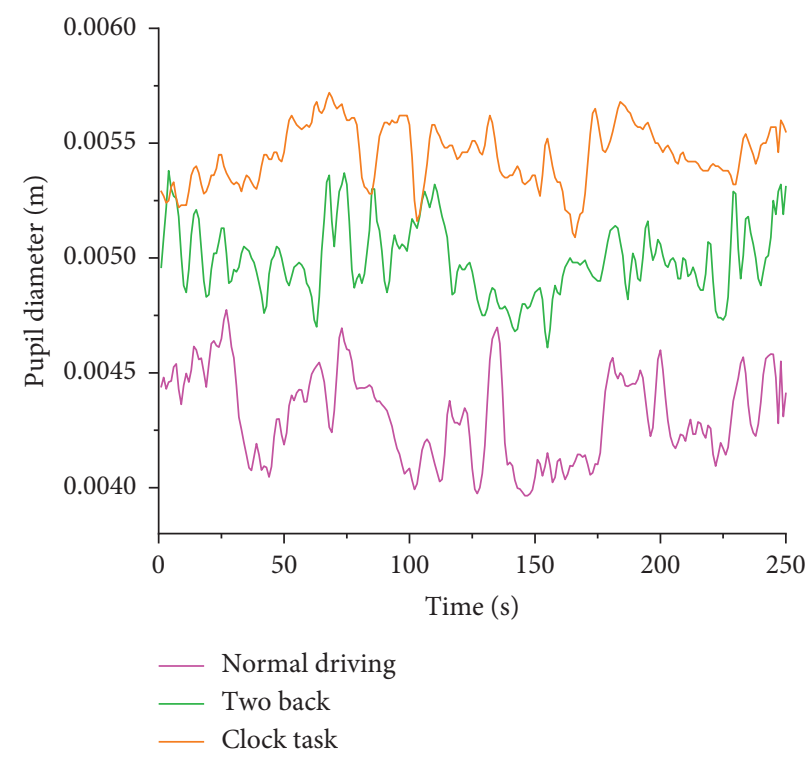

(b)

FIgURE 5: Change in pupil diameter in the (a) traditional traffic environment and (b) mixed traffic environment.

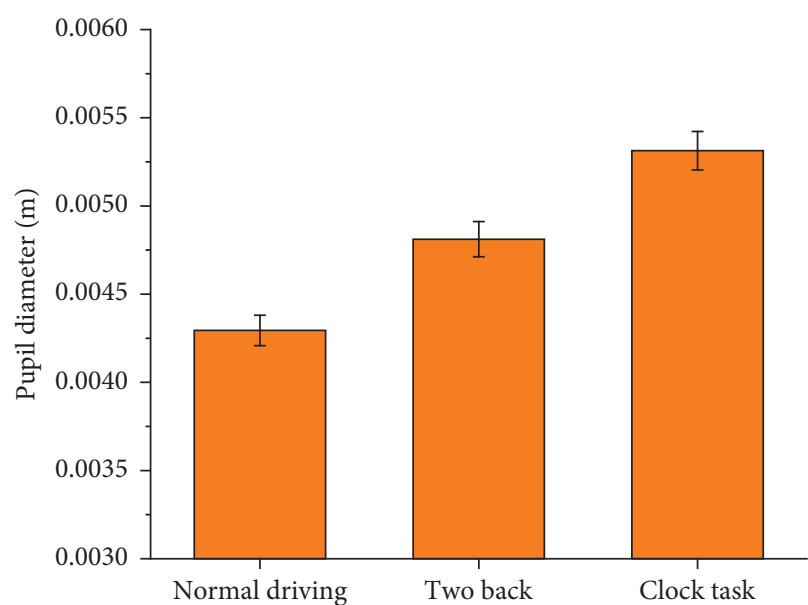

(a)

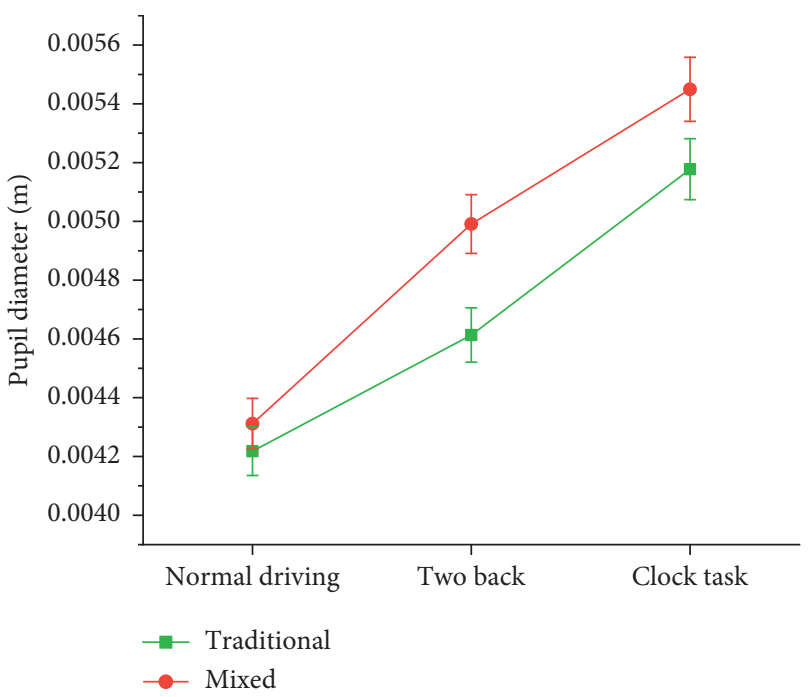

(b)

Figure 6: (a) Main effect of the driving tasks on the pupil diameter. (b) Interaction effect of the driving tasks and traffic environment on the pupil diameter.

followed by those in the two-back task. However, no significant difference exists between the clock task and twoback task.

The interaction effect of the driving task and environment on the SD of the horizontal fixation angles is significant; however, this effect is not significant for the SD of the vertical fixation angles. The post hoc test results show that the SD of the horizontal fixation angles is lower in the mixed traffic environment than in the traditional traffic environment (as shown in Figure 8(a)). Moreover, with those in the traditional traffic environment, the values of the overall baseline SDs of the vertical fixation angles in the mixed traffic environment are lower (as shown in Figure 8(b)).

3.2.3. Blink Frequency. A 2 (environment) $\times 3$ (driving task) repeated-measures ANOVA was performed for the blink frequency. The results are shown in Table 2 . The driving task and environment exert significant effects on the blink frequency. The mean blink frequency across the driving tasks is shown in Figure 9. The post hoc analysis results show that 


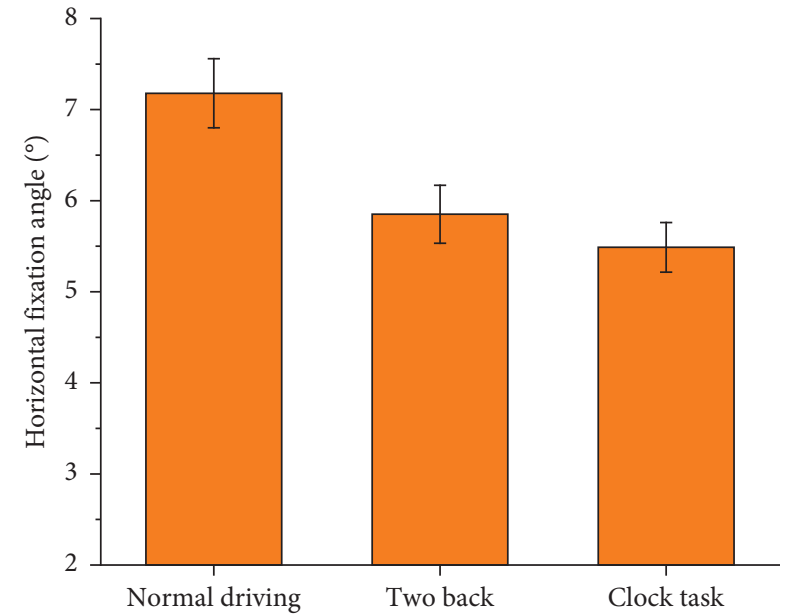

(a)

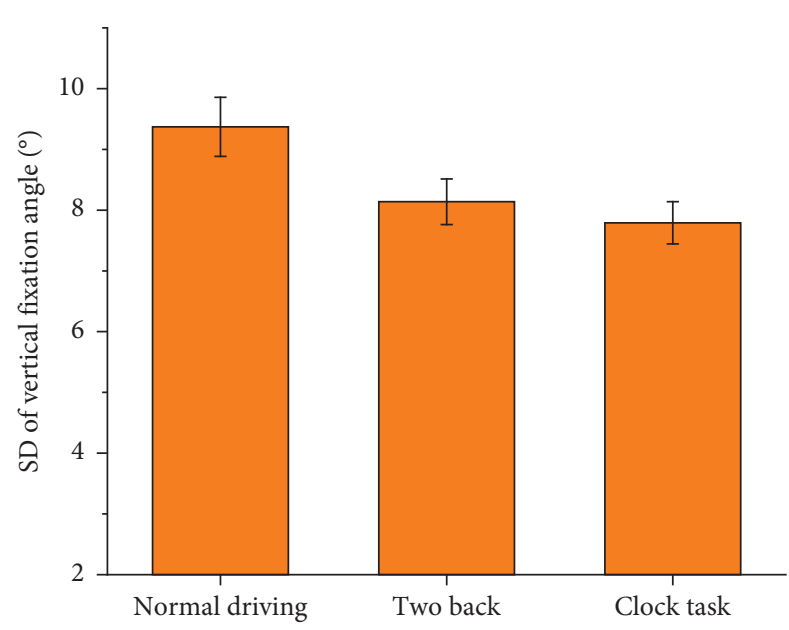

(b)

FIgURE 7: Main effect of the driving tasks on the (a) horizontal fixation angle and (b) vertical fixation angle.

the two-back task and clock task exert significant effects on the blink frequency compared to those in normal driving, and the difference between the two-back task and clock task is significant $(p=0.002)$. The interaction effect of the driving tasks with the environment is significant. The post hoc results show that the blink frequency of the drivers in each driving task in mixed traffic environments is higher than that in traditional traffic environments (as shown in Figure 10). This phenomenon can be attributed to the complexity of the mixed traffic environment. When the driver is affected by the complex traffic environment, the blink frequency increases significantly, which makes the overall baseline level higher.

3.2.4. Speed. A repeated-measures ANOVA was performed to investigate the vehicle speed under different driving tasks in the two traffic environments. The results show that the main effects of the driving tasks and environments on the speed are significant (see Table 2).

Subsequent post hoc tests were conducted, which show that the two-back task results in a significant difference in speed compared to that under normal driving. Moreover, the effect of the clock task on the speed of drivers corresponds to the largest decrease from that under normal driving $[42,43]$. A significant difference exists between the speeds associated with the two-back task and clock task. The mean speed across driving tasks is shown in Figure 11. Moreover, Table 2 shows that the interaction effect of the driving task and traffic environment is significant. The post hoc results show that drivers who performed driving tasks in traditional traffic environments drove at higher speeds than those in mixed traffic environments (as shown in Figure 12). The difference occurs because the mixed traffic environment is more complex and part of the driver's cognitive resources need to be applied to address this complexity. The driver subconsciously reduces the speed to cope with the changes in the external environment to ensure safe driving.
3.2.5. Mean and Standard Deviation (SD) of the Acceleration. Table 2 shows that the mean and SD of the acceleration of the drivers do not significantly differ across the driving tasks and traffic environments moreover, the interaction effect of the driving task and traffic environment is not significant.

According to these analysis results, the mean and SD of the longitudinal acceleration are not significantly different under the driving tasks in the two environments. The minor corrections are similar in the traditional and mixed environments. Therefore, these two parameters cannot capture a cognitively distracted driver state.

3.2.6. SDLP. The SDLP can reflect the driver's lane-keeping ability in the execution of driving tasks; this parameter was recorded in the experiment. A 2 (environment) $\times 3$ (driving task) repeated-measures ANOVA was conducted on the SDLP.

The SDLP does significantly differ across the driving tasks (see Table 2). The mean values of the SDLP across the driving tasks are shown in Figure 13. Bonferroni's post hoc tests indicate that driving under the two-back task is significantly different from normal driving, and the SDLP values for drivers who perform clock tasks are significantly higher than those under normal driving $[35,36]$. However, no significant difference exists between the SDLP values under the two-back task and clock task. Although the interaction effects of the driving task and environment are not significant (see Table 2), the SDLP exhibits a higher value in the mixed traffic environment than in the traditional traffic environment, and drivers performing the clock task exhibit the highest SDLP value in the mixed traffic environment (as shown in Figure 14).

3.2.7. Mean and SDSWA. The mean and SDSWA indicate the degree of change in the angular position of a steering wheel $[44,45]$, which can indicate the steering smoothness and instability. 


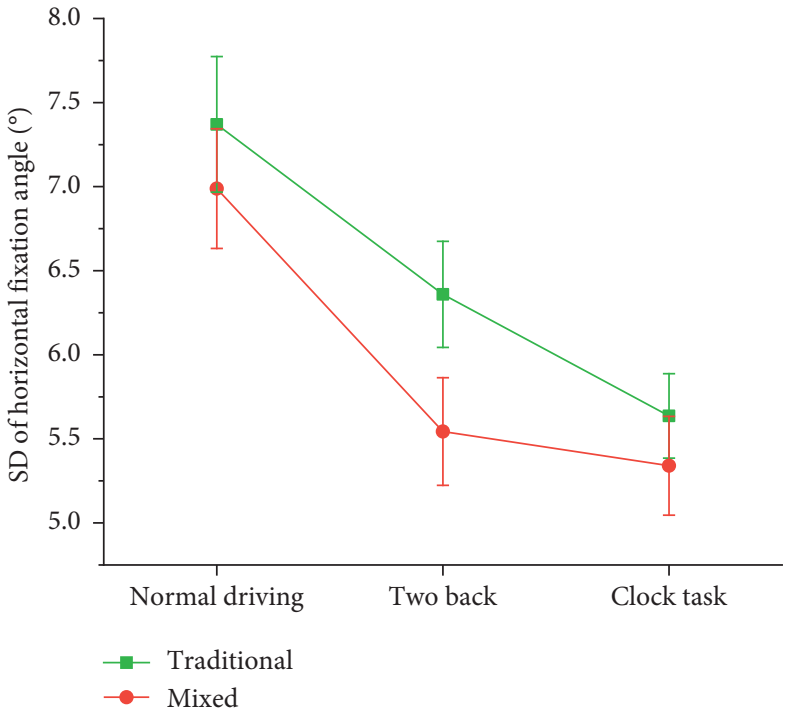

(a)

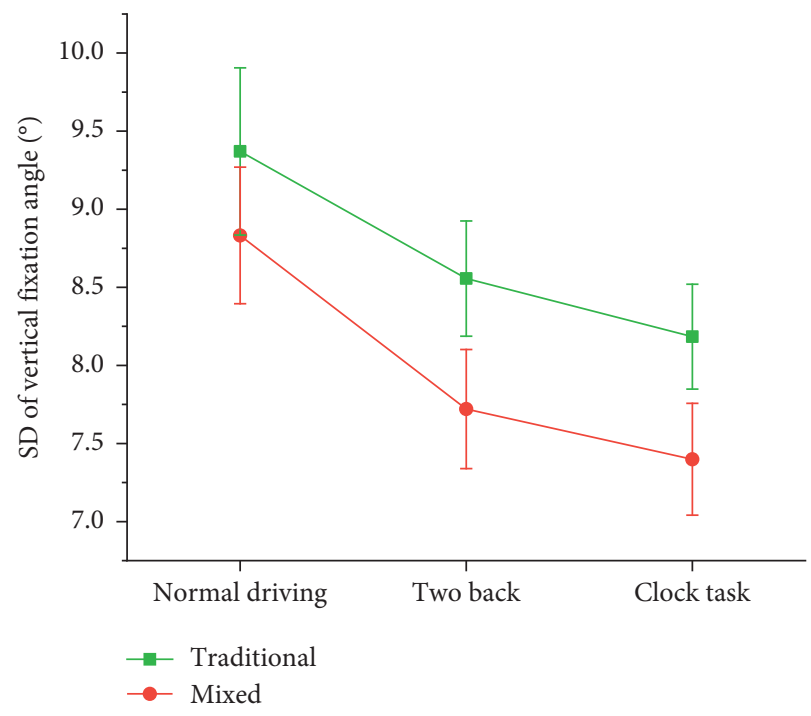

(b)

FIGURE 8: Interaction effect of driving tasks and traffic environment on the SD of the (a) horizontal fixation angle and (b) vertical fixation angle.

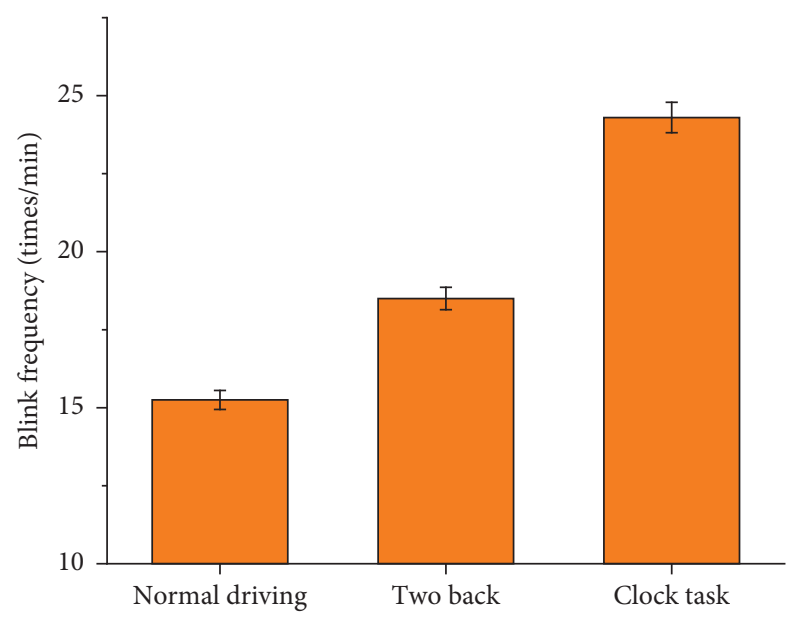

FIGURE 9: Main effect of the driving tasks on the blink frequency.

A 2 (environment) $\times 3$ (driving task) repeated-measures ANOVA was performed for the mean and SDSWA, and the results are shown in Table 2 . The analysis shows that the interaction effect of the driving task and environment on the mean steering wheel angle is not significant. Moreover, the effects of the driving tasks and environments on the mean steering wheel angles are not significant. However, the driving tasks have a significant main effect on the SDSWA, as shown in Figure 15. The post hoc test shows that, under the clock task and two-back tasks, the SDSWA is higher than that under normal driving, and the SDSWA changes significantly under distracted driving [46]. However, the results under the two-back task and clock task are not significantly different $(p=0.074)$. The interaction effect of the driving task and environment is significant on the SDSWA. When driving tasks are performed in the mixed traffic environment, the SDSWA is higher than that in the traditional traffic environment (as shown in Figure 16).

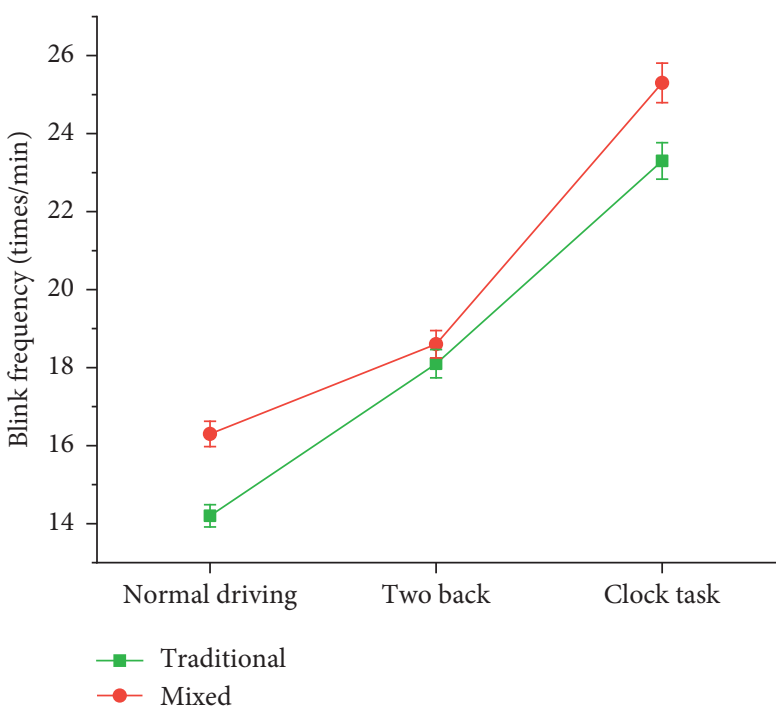

Figure 10: Interaction effect of the driving tasks and environment on the blink frequency.

3.2.8. Steering Entropy (SE). Table 2 shows that the interaction effect of the driving tasks with the environments is not significant; in contrast, the mean SE values significantly differ across the driving tasks. Bonferroni's post hoc test results show that the SE values under distracted driving and normal driving are significantly different, and the mean SE under the clock task is the highest, followed by that under the two-back task $[47,48]$. The mean SE values (with the standard error) across the driving tasks are shown in Figure 17. No significant difference can be observed between the SE values under the clock task and twoback task. Moreover, the environment does not have a significant effect. The results indicate that the influence of the driving tasks on the SE in the traditional and mixed traffic environments is similar (as shown in Figure 18). 


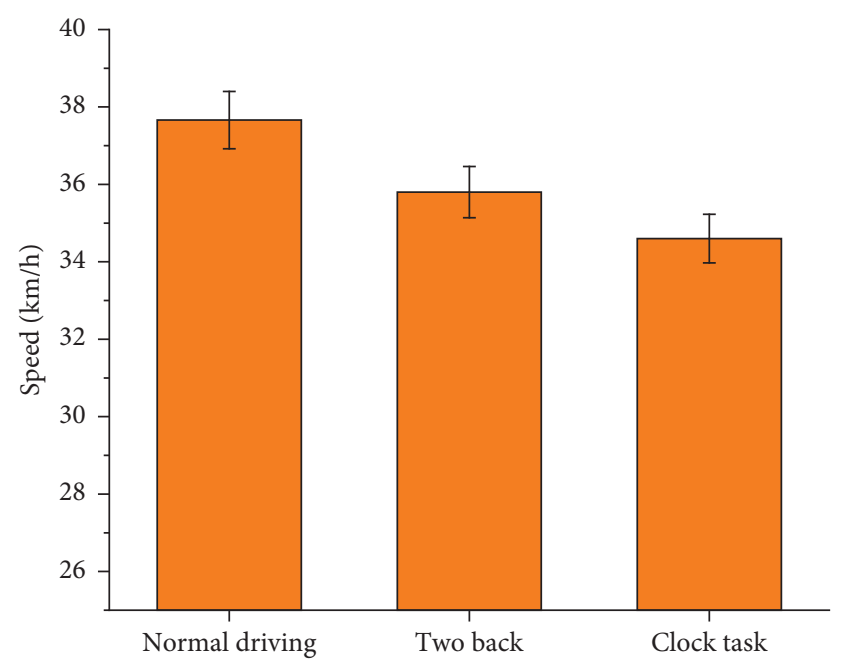

FIgURE 11: Main effect of the driving tasks on the speed.

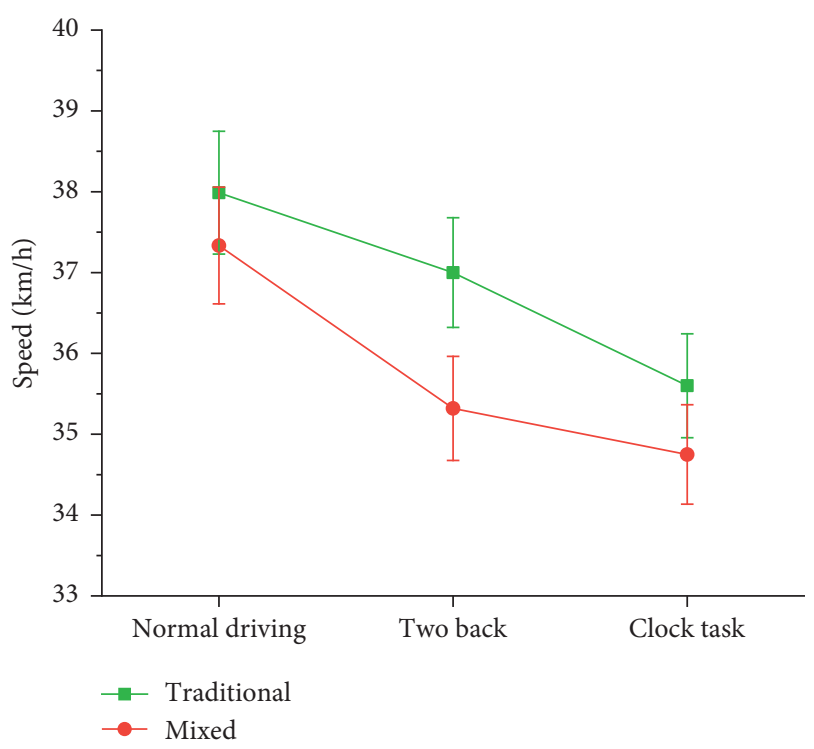

FIgURE 12: Interaction effect of the driving tasks and traffic environment on the speed.

3.2.9. Subjective Evaluation Performance. Significant relationships exist between the workload, situational awareness, and number of traffic accidents caused by distracted driving. In this paper, subjective evaluation is performed to examine the drivers' subjective feelings during the driving tasks in the two traffic environments. The subjective evaluation criteria are shown in Table 3. A higher workload score corresponds to a higher workload, and a higher situational awareness score corresponds to a higher situational awareness ability. The scoring results are shown in Table 4.

Table 2 shows that the interaction effects of the driving tasks and environments on the workload assessment performance are not significant. The results show that the trends of workload assessment performance scored by participants in traditional and mixed traffic environments

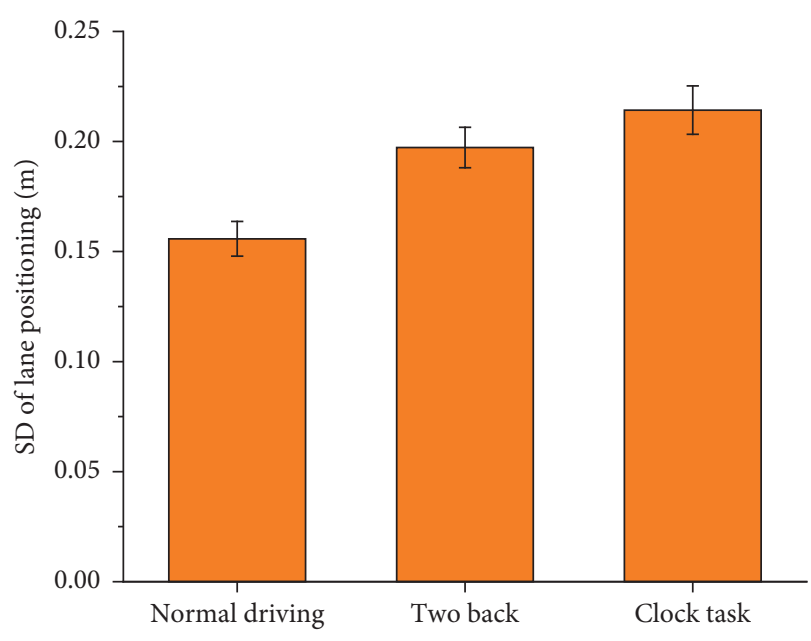

Figure 13: Main effect of the driving tasks on the SDLP.

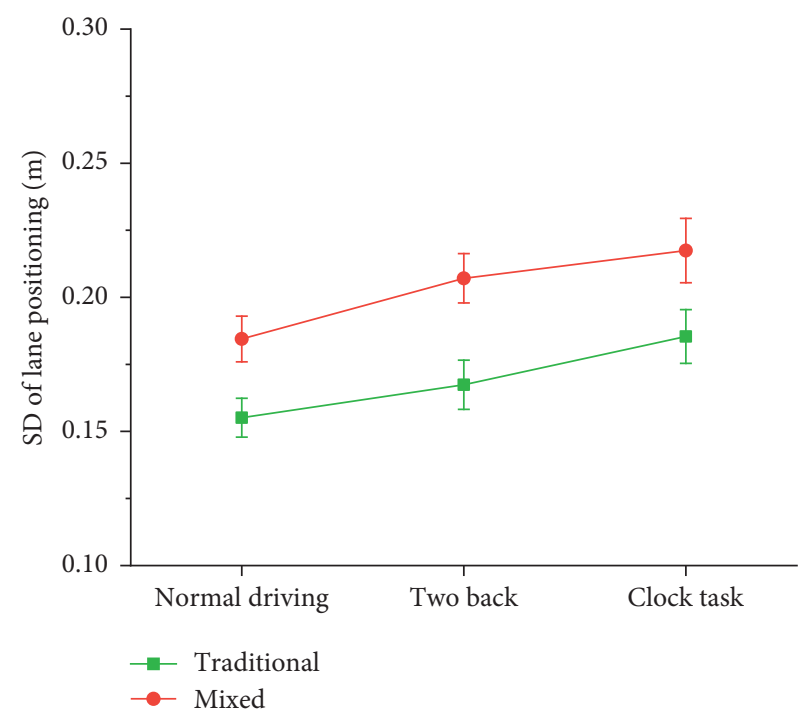

FIGURE 14: Interaction effect of the driving tasks and environment on the SDLP.

are similar (as shown in Figure 19(a)). The interaction effects of the driving tasks and environments on the environment perception performance are significant; distracted drivers in the mixed traffic environment exhibit an inferior environment perception performance compared to those in the traditional traffic environment (as shown in Figure 19(b)). The main effects of the driving tasks are significant (see Table 3 ). The mean workload assessment performance across the driving tasks is plotted in Figure 20(a).

A post hoc test was performed. The results indicate that the workload assessment performance under distracted driving (two-back task: 3.599; clock task: 3.969) and environmental perception performance under distracted driving (two-back tasks: 3.951; clock task: 3.298) (see Figure 20(b)) are significantly higher $(p<0.001)$ than those under normal driving. The results for the two-back tasks and clock tasks are also significantly different. 


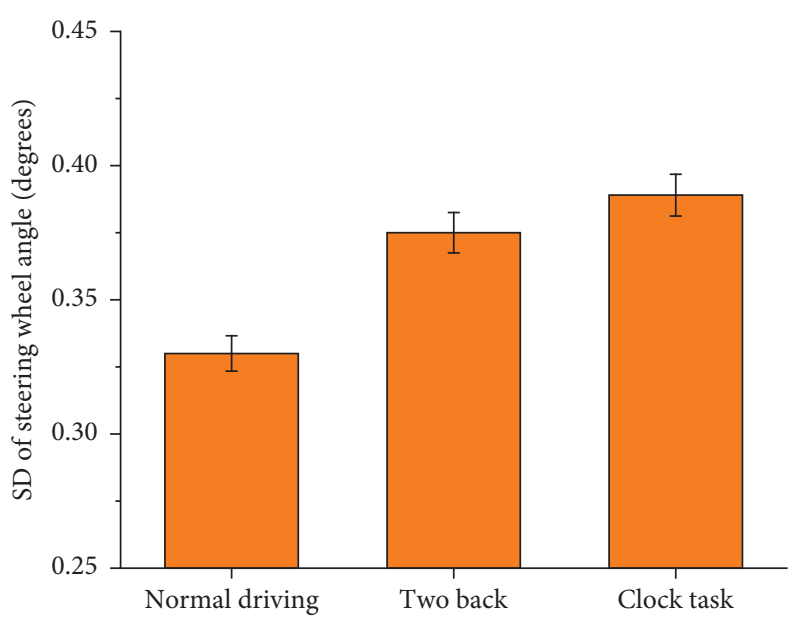

FIGURE 15: Main effect of the driving tasks on the SDSWA.

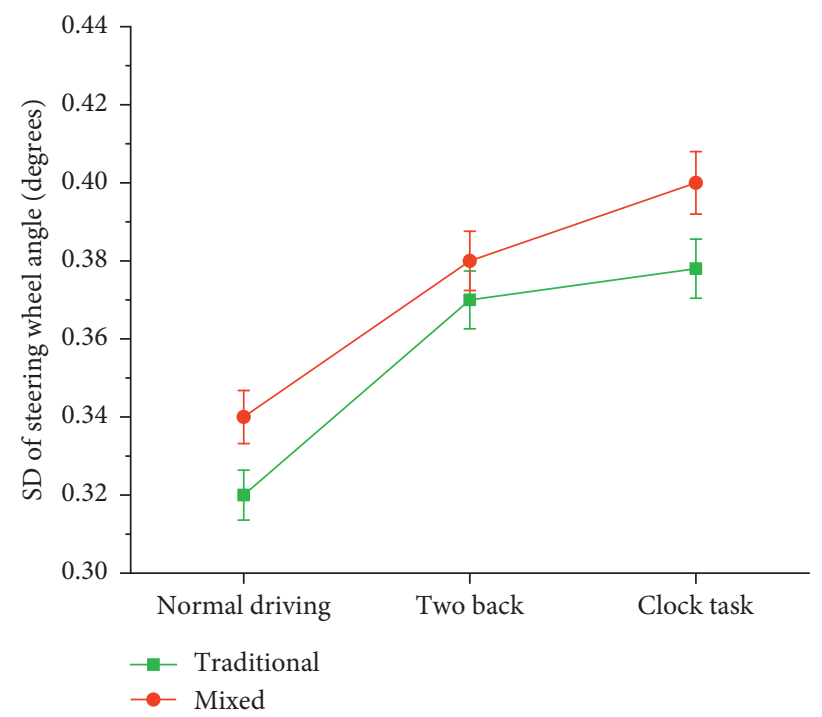

FIGURE 16: Interaction effect of the driving tasks and environment on the SDSWA.

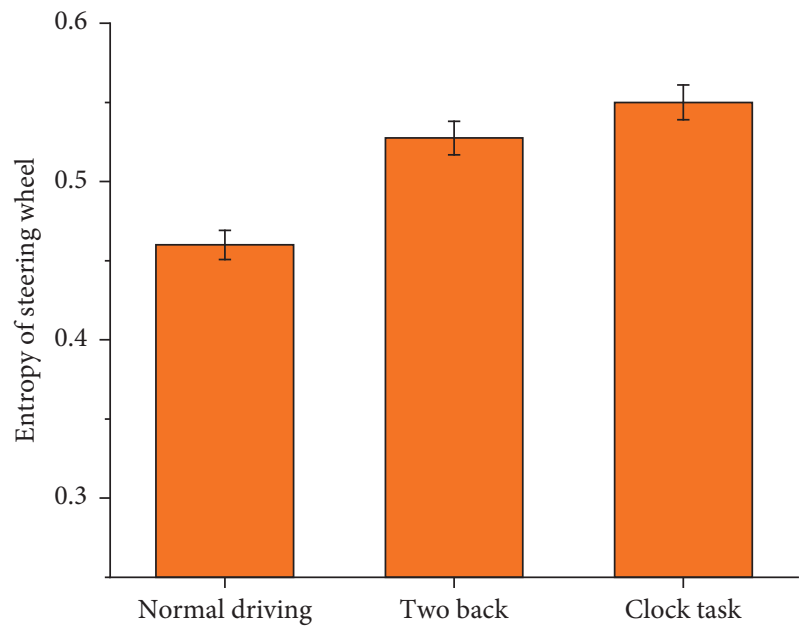

Figure 17: Main effect of the driving tasks on the SE.

\section{Discussion and Conclusion}

Sixty participants were involved in driving tasks (normal driving, two-back task, and clock task) in traditional and mixed traffic environments. The following driver physiological measures, driving performance parameters, and subjective performance were considered: pupil diameter, fixation angle, blink frequency, speed, mean acceleration, SD of acceleration, SDLP, mean steering wheel angle, SDSWA, SE, workload assessment performance, and environmental perception performance. The main independent variables were three different driving tasks (normal driving, two-back task, and clock task), and the two traffic environments were traditional and mixed traffic environments. Moreover, the main effects of the driving tasks and environments on these performance parameters were analysed. The interaction effects of the variables were investigated by performing mixed design repeated-measures ANOVA tests and 


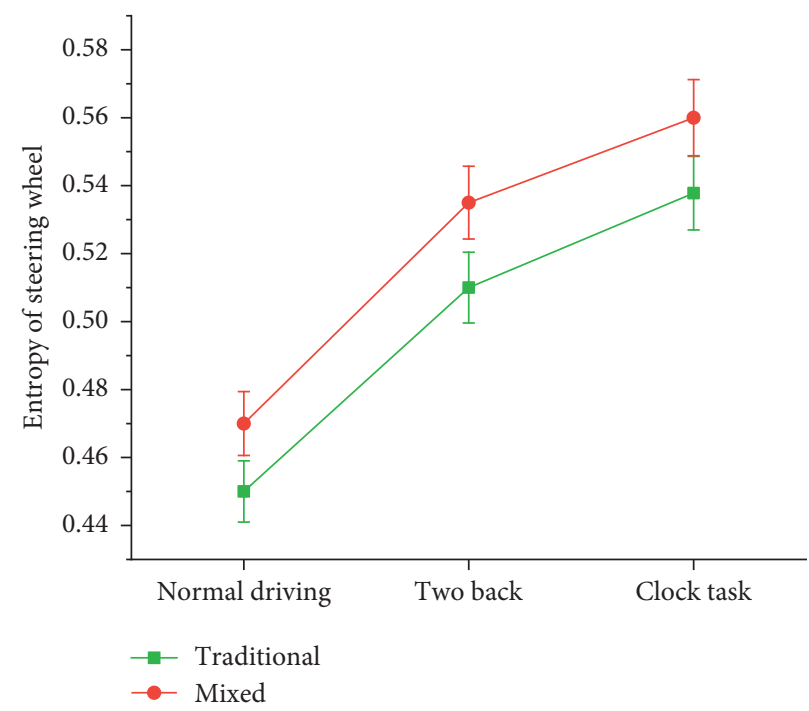

Figure 18: Interaction effect of the driving tasks and environment on the SE.

TABLE 3: Subjective evaluation criteria.

\begin{tabular}{lccccccc}
\hline Scoring item & 0 & 1 & 2 & 3 & 4 & 5 & 6 \\
\hline Workload assessment & Extremely low & - & - & - & - & - & Extremely high \\
Environmental perception & Extremely low & - & - & - & - & - & Extremely high \\
\hline
\end{tabular}

TABle 4: Results.

\begin{tabular}{lcccc}
\hline Type & Scoring item & Normal driving & Two-back & Clock task \\
\hline \multirow{2}{*}{ Traditional } & Workload assessment & 1.808 & 3.474 & 3.70 \\
\multirow{2}{*}{ Mixed } & Environmental perception & 4.523 & 4.232 & 3.736 \\
& Workload assessment & 2.062 & 3.725 & 3.238 \\
& Environmental perception & 4.847 & 3.67 & 2.86 \\
\hline
\end{tabular}

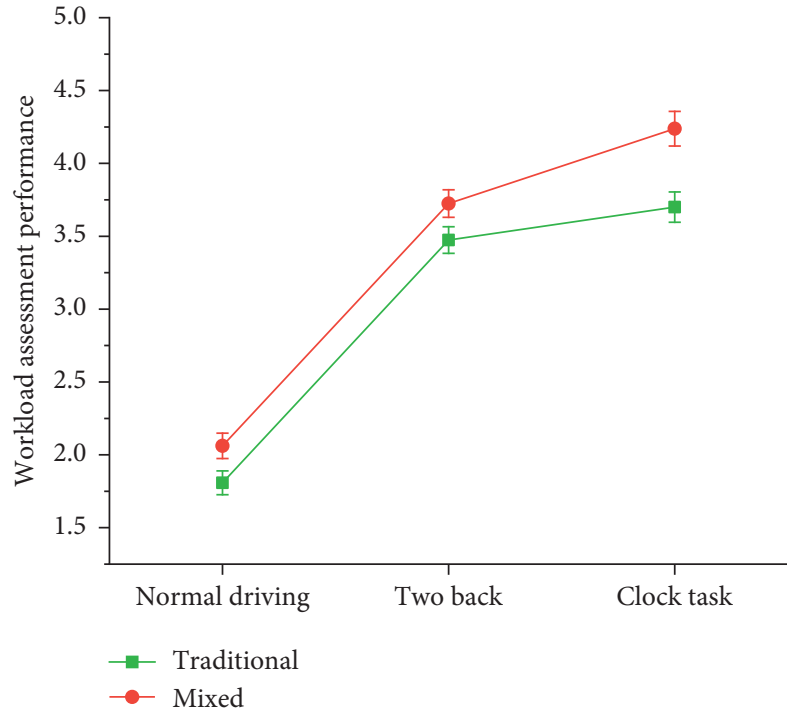

(a)

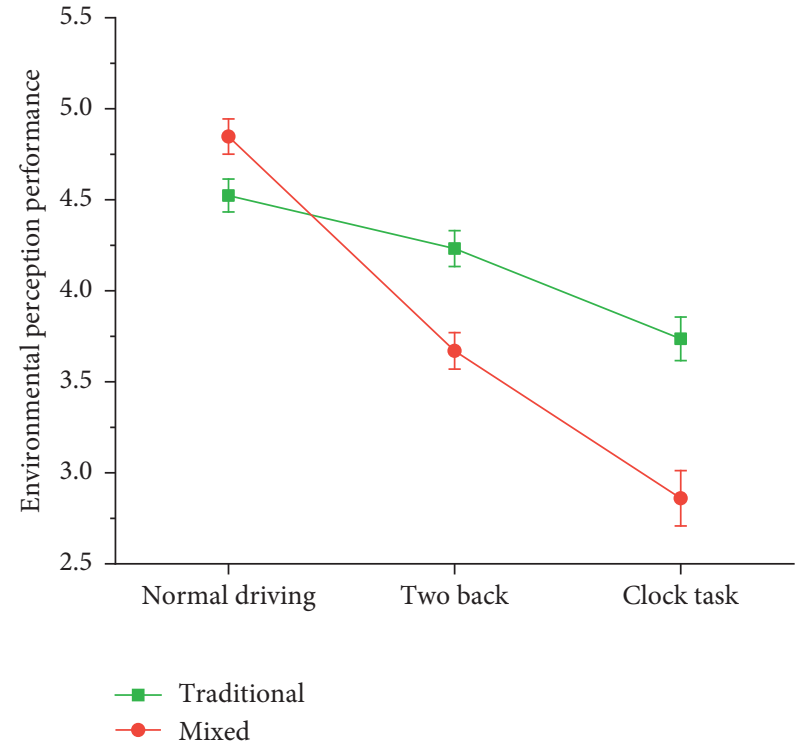

(b)

FIGURE 19: Interaction effect of the driving tasks and environment on the (a) workload assessment performance and (b) environmental perception performance. 


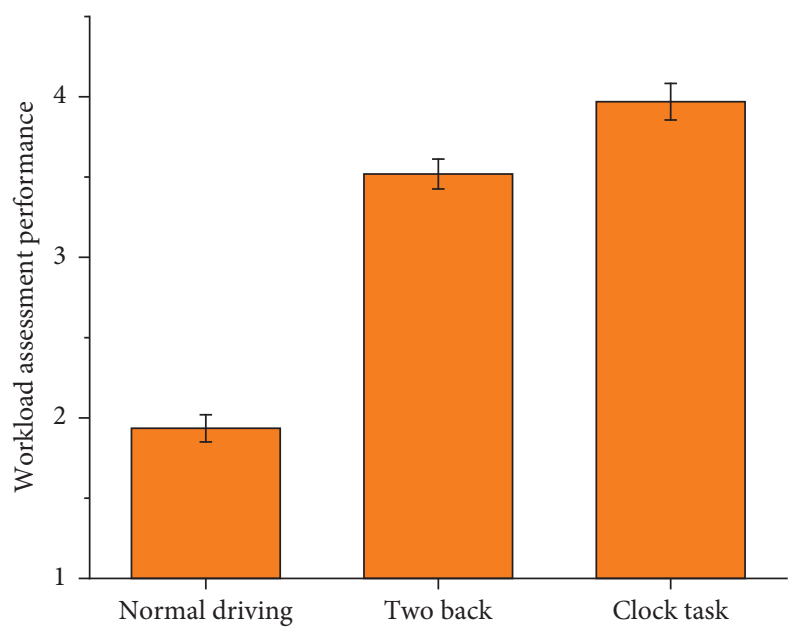

(a)

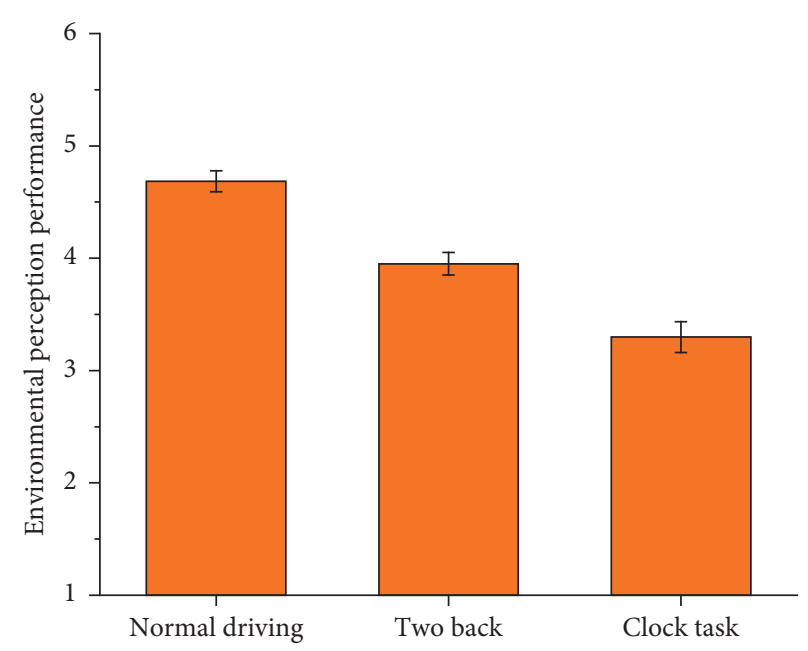

(b)

FIGURE 20: Main effect of the driving tasks on the (a) workload assessment performance and (b) environmental perception performance.

Bonferroni's post hoc tests at the 0.05 significance level. Table 5 summarizes the key observed effects of the driving tasks on these parameters in traditional and mixed traffic environments.

The results show that each of the driver physiological measures exhibits significant differences across the driving tasks. The pupil diameter and blink frequency under distracted driving are higher than those during normal driving in the two traffic environments, and these two driver physiological measures are higher in the driving tasks in the mixed traffic environment than in the traditional traffic environment. This phenomenon occurs because the driver's cognitive load increases in a mixed traffic environment, which stimulates the sympathetic nervous system. This aspect causes the dilation of the pupils and increases the blink frequency, leading to an increase in the baselines of these two metrics. However, the SD values of the horizontal and vertical fixation angles are significantly lower during distracted driving than those during normal driving in the two traffic environments. The baseline of these measures in the mixed environment is lower than that in the traditional traffic environment. A potential explanation for this aspect is that the driver's cognitive load increases in a mixed traffic environment. Moreover, in this environment, the driver's field of view is narrower than that in a traditional traffic environment and relatively concentrated in the centre of the road under distracted driving $[49,50]$. Consequently, the perception of things on both sides of the road is reduced, and conflict with lateral pedestrians is prolonged. The driver physiological measures during distracted driving are significantly different from those during normal driving in traditional and mixed traffic environments. Thus, these driver physiological measures can be considered parameters for detecting the cognitive distraction of drivers in the two traffic environments.

Furthermore, the results indicate that several driving performance parameters, such as speed, SDLP, SDSWA, and SE, are significantly different under distracted driving and normal driving in the two traffic environments. In addition, the distracting tasks that require a higher workload contribute to a higher SDLP, SDSWA, and SE and a lower speed in the two traffic environments. The result of a higher SDLP is not in line with the previously reported results [51], which may be caused by the different experimental conditions. According to the analysis results, the overall baseline of the effect of the driving tasks on these parameters in the mixed environment is stronger than that in the traditional environment, except for the speed parameter. The mean speed of drivers during driving tasks in a mixed traffic environment is lower than that in a traditional traffic environment because mixed traffic environments are complicated and occupy a part of the driver's cognitive resources. Consequently, drivers subconsciously reduce the speed of their vehicles to cope with the changes in the external environment to ensure safe driving. Moreover, when driving while performing distracting tasks, the driver's demand for cognitive resources increases. Thus, the driver actively reduces the driving speed, which can be regarded as a compensation effect. When the driver performs driving tasks in a mixed environment, especially distracting tasks, the baseline values of the SDLP, SDSWA, and SE are higher than those in a traditional traffic environment, which can explain why drivers need extensive cognitive resources in a mixed traffic environment. Additionally, the number of corrections to the steering wheel by the driver is reduced, leading to the aggravation of these parameters. Unlike normal driving, the effects of certain parameters, including the SDLP, SDSWA, and SE, are significant under distracted driving, and thus, these parameters can be considered candidate parameters for distraction recognition in the two traffic environments. The mean and SD of acceleration and mean steering wheel angle exhibit no significant effects across driving tasks and traffic environments and therefore cannot be applied as parameters to measure cognitive distraction. 
TABLE 5: Results of analyses of driver physiological measures and driving performance parameters.

\begin{tabular}{|c|c|c|c|c|c|}
\hline Independent variable & & Mi vs $\operatorname{Tr}$ & T vs $\mathrm{N}$ & C vs $\mathrm{N}$ & $\mathrm{C}$ vs $\mathrm{T}$ \\
\hline Pupil diameter & & Increased* $\uparrow$ & Increased* $\uparrow$ & Increased $^{*} \uparrow$ & Increased* $\uparrow$ \\
\hline \multirow{2}{*}{ Fixation angle } & Horizontal & Decreased & Decreased $^{*} \downarrow$ & Decreased $^{*} \downarrow$ & Decreased \\
\hline & Vertical & Decreased $^{*} \downarrow$ & Decreased* $\downarrow$ & Decreased $^{*} \downarrow$ & Decreased \\
\hline \multirow{2}{*}{$\begin{array}{l}\text { Blink frequency } \\
\text { Speed }\end{array}$} & & Increased ${ }^{*} \uparrow$ & Increased ${ }^{*} \uparrow$ & Increased $^{*} \uparrow$ & Increased $* \uparrow$ \\
\hline & & Decreased* ${ }^{*} \downarrow$ & Decreased* ${ }^{*} \downarrow$ & Decreased $^{*} \downarrow$ & Decreased $^{*} \downarrow$ \\
\hline \multirow{2}{*}{ Acceleration } & Mean & - & - & - & - \\
\hline & SD & - & - & - & - \\
\hline SDLP & & Increased & Increased ${ }^{*} \uparrow$ & Increased ${ }^{*} \uparrow$ & Increased \\
\hline Mean steering wheel angle & - & - & - & - & \\
\hline SDSWA & & Increased & Increased* $\uparrow$ & Increased ${ }^{*} \uparrow$ & Increased \\
\hline SE & & Increased & Increased ${ }^{*} \uparrow$ & Increased ${ }^{*} \uparrow$ & Increased \\
\hline \multirow{2}{*}{ Subjective performance } & Workload assessment & Increased & Increased ${ }^{*} \uparrow$ & Increased $^{*} \uparrow$ & Increased* $\uparrow$ \\
\hline & Environment perception & Decreased* $\downarrow$ & Decreased $^{*} \downarrow$ & Decreased $^{*} \downarrow$ & Decreased $^{*} \downarrow$ \\
\hline
\end{tabular}

$\operatorname{Tr}=$ traditional traffic environment, $\mathrm{Mi}=$ mixed traffic environment, $N=$ normal driving, $T=$ two-back task, $C=$ clock task, decreased ${ }^{*} \downarrow=$ significant, increased $^{*} \uparrow=$ significant, decreased $=$ insignificant, and increased $=$ insignificant.

Interestingly, driving tasks exert a significant effect on the environmental perception performance and workload assessment performance, with the environmental perception performance being inversely proportional to workload assessment performance. Distracting tasks incur a higher driver cognitive load than normal driving, and the clock task consumes the largest load. In contrast, the environment perception ability is the most inferior in the clock task, followed by that in the two-back tasks. The results indicate that drivers experience a higher cognitive load in a mixed traffic environment for driving tasks than that in a traditional environment, and the environmental perception ability, affected by the distracting tasks, is lower in a mixed traffic environment than that in a traditional traffic environment. This phenomenon can be attributed to the interaction effect between the distracting tasks and environment. Although the development of intelligent transportation has enhanced the convenience for human beings, it is accompanied by certain uncertain factors. The subjective questionnaire survey shows that distractions in a mixed traffic environment are highly dangerous.

Distracted driving in traditional and mixed traffic environments can reduce the drivers' ability to perceive sudden traffic events. Therefore, it is particularly important to install a distraction recognition system on intelligent vehicles to reduce the occurrence probability of traffic accidents. In this study, the abovementioned findings can be used to understand the driver's driving experience in a mixed traffic environment and identify the differences in the distractions between traditional traffic and mixed environments. The differences between the driver's physiological measures and the driving performance parameters under these distracted driving behaviours provide a theoretical basis for the study of cognitive distraction recognition models in two traffic environments. The impacts of distracted driving on drivers' physiological measures, driving performance parameters, and subjective evaluation performance in traditional and mixed environments are analysed to provide a valuable reference to identify the most appropriate parameters for distraction recognition.
The research in this paper still has some shortcomings. First, similar to several previous studies, the experiments were conducted in a simulated driving environment by using a driving simulator, which lacks the realism of the traffic environment. Future work can be aimed at investigating the distraction effects on parameters collected in a real traditional and mixed traffic environment. Second, the sample size of drivers in this study was small (60), and future work must be aimed at considering a large sample. Third, measures to produce distracted driving were realized through secondary tasks. In future work, more realistic tasks, such as those pertaining to distracting behaviours that often occur in daily life, must be adopted.

\section{Data Availability}

The data used to support the findings of this study are available from the corresponding author upon request.

\section{Conflicts of Interest}

The authors declare that they have no conflicts of interest.

\section{Authors' Contributions}

Q. Hua contributed to the methodology, data analysis, and writing the original draft. L. Jin contributed to establishing the experimental platform and providing financial support. Y. Jiang contributed to conducting the experiments. B. Guo and $\mathrm{X}$. Xie contributed to enhancing the manuscript.

\section{Acknowledgments}

This work was supported by the National Key Research and Development Program of China (Grant 2018YFB1600501), National Natural Science Foundation of China (nos. U19A2069 and 52072333), Natural Science Foundation of Hebei Province (no. E2020203092), and Hebei Provincial Key Research Projects (no. 20310801D). 


\section{References}

[1] WHO fact sheets, May 2014: The Top 10 Causes of Death, 2016, http://www.who.int/mediacentre/factsheets/fs310/en/.

[2] National Highway Traffic and Safety Administration [NHTSA], Distracted Driving 2015 Traffic Safety Facts Research Note, U. S. Department of Transportation, Washington, DC, USA, 2017.

[3] H.-P. Shao, J. Yin, W.-H. Yu, and Q.-L. Wang, "Aberrant driving behaviours on risk involvement among drivers in China," Journal of Advanced Transportation, vol. 2020, pp. 1-8, 2020.

[4] S. Shen and D. M. Neyens, "Factors affecting teen drivers' crash-related length of stay in the hospital," Journal of Transport and Health, vol. 4, pp. 162-170, 2017.

[5] W. Do, O. M. Rouhani, and L. Miranda-Moreno, "Simulation-based connected and automated vehicle models on highway sections: a literature review," Journal of Advanced Transportation, vol. 2019, pp. 1-14, 2019.

[6] Y. Xing, C. Lv, X. Mo, Z. Hu, C. Huang, and P. Hang, “Toward safe and smart mobility: energy-aware deep learning for driving behavior analysis and prediction of connected vehicles," IEEE Transactions on Intelligent Transportation Systems, vol. 99, pp. 1-14, 2021.

[7] K. Shaaban, "Drivers' perceptions of smartphone applications for real-time route planning and distracted driving prevention," Journal of Advanced Transportation, vol. 2019, no. 1453, pp. 1-10, 2019.

[8] D. Tran, H. Manh Do, W. Sheng, H. Bai, and G. Chowdhary, "Real-time detection of distracted driving based on deep learning," IET Intelligent Transport Systems, vol. 12, no. 10, pp. 1210-1219, 2018.

[9] H. Saenz, H. Sun, L. Wu, X. Zhou, and H. Yu, "Detecting phone-related pedestrian distracted behaviours via a twobranch convolutional neural network," IET Intelligent Transport Systems, vol. 15, no. 2, pp. 147-158, 2020.

[10] Y. Liang and J. D. Lee, "A hybrid bayesian network approach to detect driver cognitive distraction," Transportation Research Part C: Emerging Technologies, vol. 38, no. 1, pp. 146-155, 2014.

[11] M. Miyaji, H. Kawanaka, and K. Oguri, "Effect of pattern recognition features on detection for driver's cognitive distraction," in Proceedings of 13th International IEEE Conference on Intelligent Transportation Systems, Funchal, Portugal, September 2010.

[12] Y. Liang, "Detecting driver distraction," Diss. Theses Gradworks, 2009.

[13] J. D. Mckeever, M. T. Schultheis, V. Padmanaban, and A. Blasco, "Driver performance while texting: even a little is too much," Traffic Injury Prevention, vol. 14, no. 2, pp. 132-137, 2013.

[14] R. Thapa, J. Codjoe, S. Ishak, and K. S. Mccarter, "Post and during event effect of cell phone talking and texting on driving performance-A driving simulator study," Traffic Injury Prevention, vol. 16, no. 5, pp. 461-467, 2015.

[15] C. Irwin, S. Monement, and B. Desbrow, "The influence of drinking, texting, and eating on simulated driving performance," Traffic Injury Prevention, vol. 16, no. 2, pp. 116-123, 2015.

[16] Y. K. Ou, "Effects of age and the use of hands-free cellular phones on driving behavior and task performance," Traffic Injury Prevention, vol. 12, no. 6, pp. 550-558, 2011.

[17] Z. Chen, C. Wu, M. Zhong, N. Lyu, and Z. Huang, "Identification of common features of vehicle motion under drowsy/distracted driving: a case study in Wuhan, China," Accident Analysis \& Prevention, vol. 81, pp. 251-259, 2015.

[18] L. Yuan, S. E. Li, W. Wang, W. Ying, and C. Bo, "The impact of driver cognitive distraction on vehicle performance at stopcontrolled intersections," in Proceedings of 13th International IEEE Conference on Intelligent Vehicles Symposium (IV), vol. 6, Seoul, Korea, June 2015.

[19] J. K. Engelberg, L. L. Hill, J. Rybar, and T. Styer, "Distracted driving behaviors related to cell phone use among middleaged adults," Journal of Transport and Health, vol. 2, no. 3, pp. 434-440, 2015.

[20] K. Shaaban, S. Gaweesh, and M. M. Ahmed, "Characteristics and mitigation strategies for cell phone use while driving among young drivers in Qatar," Journal of Transport and Health, vol. 8, pp. 6-14, 2018.

[21] S. Bovonsunthonchai, R. Ariyaudomkit, T. E. Susilo, P. Sangiamwong, and J. Richards, "The impact of different mobile phone tasks on gait behaviour in healthy young adults," Journal of Transport and Health, vol. 19, Article ID 100920, 2020.

[22] P. Choudhary and N. R. Velaga, "Analysis of vehicle-based lateral performance measures during distracted driving due to phone use," Transportation Research Part F: Traffic Psychology and Behaviour, vol. 44, pp. 120-133, 2017.

[23] B. Metz, A. Landau, and V. Hargutt, "Frequency and impact of hands-free telephoning while driving - results from naturalistic driving data," Transportation Research Part F: Traffic Psychology and Behaviour, vol. 29, pp. 1-13, 2015.

[24] J. K. Caird, K. A. Johnston, C. R. Willness, M. Asbridge, and P. Steel, "A meta-analysis of the effects of texting on driving," Accident Analysis and Prevention, vol. 71, no. 10, pp. 311-318, 2014.

[25] G. Fancello, M. Adamu, P. Serra, and P. Fadda, "Comparative analysis of the effects of mobile phone use on driving performance using anova and ancova," IET Intelligent Transport Systems, vol. 14, no. 9, pp. 993-1003, 2020.

[26] G. Yannis, A. Laiou, P. Papantoniou, and C. Christoforou, "Impact of texting on young drivers' behavior and safety on urban and rural roads through a simulation experiment," Journal of Safety Research, vol. 49, no. 25, pp. 25-31, 2014.

[27] F. Zong and Z. He, "Bus-car mode identification: a traffic condition-based random forests method," Journal of Transportation Engineering, vol. 146, no. 10, 2020.

[28] X. Chen, Z. Li, Y. Yang, L. Qi, and R. Ke, "High-resolution vehicle trajectory extraction and denoising from aerial videos," IEEE Transactions on Intelligent Transportation Systems, vol. 99, pp. 1-13, 2020.

[29] B. W. Weaver and P. R. Delucia, "A systematic review and meta-analysis of takeover performance during conditionally automated driving," Human Factors The Journal of the $\mathrm{Hu}$ man Factors and Ergonomics Society, 2020.

[30] B. Zhang, J. de Winter, S. Varotto, R. Happee, and M. Martens, "Determinants of take-over time from automated driving: a meta-analysis of 129 studies," Transportation Research Part F: Traffic Psychology and Behaviour, vol. 64, pp. 285-307, 2019.

[31] S.-A. Kaye, S. Demmel, O. Oviedo-Trespalacios, W. Griffin, and I. Lewis, "Young drivers' takeover time in a conditional automated vehicle: the effects of hand-held mobile phone use and future intentions to use automated vehicles," Transportation Research Part F: Traffic Psychology and Behaviour, vol. 78, no. 2, pp. 16-29, 2021.

[32] Y. Wang, B. Reimer, J. Dobres, and B. Mehler, "The sensitivity of different methodologies for characterizing drivers' gaze 
concentration under increased cognitive demand," Transportation Research Part F: Traffic Psychology and Behaviour, vol. 26, pp. 227-237, 2014.

[33] G. Underwood, D. Crundall, and P. Chapman, "Selective searching while driving: the role of experience in hazard detection and general surveillance," Ergonomics, vol. 45, no. 1, pp. 1-12, 2002.

[34] O. Nakayama, T. Futami, T. Nakamura, and E. R. Boer, "Development of a steering entropy method for evaluating driver workload," SAE Technical Paper, 1999, 1999-01-0892.

[35] K. E. Beede and S. J. Kass, "Engrossed in conversation: the impact of cell phones on simulated driving performance," Accident Analysis and Prevention, vol. 38, no. 2, pp. 415-421, 2006.

[36] N. Tractinsky, E. S. Ram, and D. Shinar, "To call or not to callThat is the question (while driving)," Accident Analysis and Prevention, vol. 56, pp. 59-70, 2013.

[37] D. Kahneman, A. Crider, D. Shapiro, and B. Tursky, "Pupillary heart rate and skin resistance changes during a mental task," Journal of Experimental Psychology, vol. 79, no. 1, pp. 164-167, 1969.

[38] J.-H. Kim and W.-S. Lee, "Effect of driver's cognitive distraction on driver's physiological state and driving performance," Journal of the Ergonomics Society of Korea, vol. 31, no. 2, pp. 371-377, 2012.

[39] G. K. Kountouriotis and N. Merat, "Leading to distraction: driver distraction, lead car, and road environment," Accident Analysis and Prevention, vol. 89, pp. 22-30, 2016.

[40] J. Engstrström, E. Johansson, and J. Östlund, "Effects of visual and cognitive load in real and simulated motorway driving," Transportation. Research Part F: Traffic Psychology and Behaviour, vol. 8, no. 2, pp. 97-120, 2005.

[41] M. Kutila, M. Jokela, G. Markkula, and M. R. Rue, "Driver distraction detection with a camera vision system," in Proceedings of IEEE International Conference on Image Processing, vol. 6, San Antonio, TX, USA, September 2007.

[42] G. M. Fitch, S. A. Soccolich, G. Feng, J. Mcclafferty, and T. A. Dingus, "The impact of hand-held and hands-free cell phone use on driving performance and safety-critical event risk," Driver Performance, 2013.

[43] P. Choudhary and N. R. Velaga, "Mobile phone use during driving: effects on speed and effectiveness of driver compensatory behaviour," Accident Analysis and Prevention, vol. 106, pp. 370-378, 2017.

[44] S. Cloete and G. Wallis, "Visuomotor control of steering: the artefact of the matter," Experimental Brain Research, vol. 208, no. 4, pp. 475-489, 2011.

[45] J2944, S.A.E., "Operational definitions of driving performance measures and statistics," Society of Automotive Engineers, 2015.

[46] T. M. Garrison and C. C. Williams, "Impact of relevance and distraction on driving performance and visual attention in a simulated driving environment," Applied Cognitive Psychology, vol. 27, no. 3, pp. 396-405, 2013.

[47] B. Donmez, L. N. Boyle, and J. D. Lee, "Safety implications of providing real-time feedback to distracted drivers," Accident Analysis and Prevention, vol. 39, no. 3, pp. 581-590, 2007.

[48] A. Paul, L. N. Boyle, ER. Boer, J. Tippin, and M. Rizzo, "Steering entropy changes as a function of microsleeps," in Proceedings of 3rd International Driving Symposium on $\mathrm{Hu}$ man Factors in Driver Assessment, Training, and Vehicle Design, pp. 441-447, Public Policy Center, University of Iowa, Iowa City, IA, USA, June 2005.
[49] R. M. Wilkie, G. K. Kountouriotis, N. Merat, and J. P. Wann, "Using vision to control locomotion: looking where you want to go," Experimental Brain Research, vol. 204, no. 4, pp. 539-547, 2010.

[50] G. K. Kountouriotis, R. M. Wilkie, P. H. Gardner, and N. Merat, "Looking and thinking when driving: the impact of gaze and cognitive load on steering," Transportation Research Part F: Traffic Psychology and Behaviour, vol. 34, pp. 108-121, 2015.

[51] J. M. Cooper, N. Medeiros-Ward, and D. L. Strayer, "The impact of eye movements and cognitive workload on lateral position variability in driving," Human Factors: The Journal of the Human Factors and Ergonomics Society, vol. 55, no. 5, pp. 1001-1014, 2013. 